\title{
The structural and functional connectivity neural underpinnings of body image
}

\author{
Massieh Moayedi $^{1}$ (c) | Nasim Noroozbahari ${ }^{1}$ | Georgia Hadjis $^{1}$ | \\ Kristy Themelis $^{2,3}$ | Tim V. Salomons ${ }^{4,5}$ | Roger Newport ${ }^{2,6}$ | Jennifer S. Lewis ${ }^{7,8}$
}

${ }^{1}$ Centre for Multimodal Sensorimotor and Pain Research, Faculty of Dentistry, University of Toronto, Toronto, Ontario, Canada

${ }^{2}$ School of Psychology, University of Nottingham, Nottingham, UK

${ }^{3}$ Department of Psychology, University of Warwick, Coventry, UK

${ }^{4}$ School of Psychology and Clinical Language Sciences, University of Reading, Reading, UK

${ }^{5}$ Department of Psychology, Queen's University, Kingston, Ontario, Canada

${ }^{6}$ School of Sport, Exercise and Health Sciences, Loughborough University, Loughborough, UK

${ }^{7}$ CRPS/CCLER Service, Royal United Hospitals Bath NHS Trust, Bath

${ }^{8}$ School of Health and Social Wellbeing, Faculty of Health and Applied Sciences, Glenside Campus, University of the West of England, Bristol

\section{Correspondence}

Jennifer Lewis, School of Health and Social Wellbeing, Faculty of Health and Applied Sciences, Glenside Campus, University of the West of England, Blackberry Hill, Stapleton, Bristol BS16 1DD, UK.

Email: jenny4.lewis@uwe.ac.uk

Funding information

University of the West of England, Grant/

Award Number: 201415/31

\begin{abstract}
How we perceive our bodies is fundamental to our self-consciousness and our experience in the world. There are two types of interrelated internal body representations-a subjective experience of the position of a limb in space (body schema) and the subjective experience of the shape and size of the limb (body image). Body schema has been extensively studied, but there is no evidence of the brain structure and network dynamics underpinning body image. Here, we provide the first evidence for the extrastriate body area (EBA), a multisensory brain area, as the structural and functional neural substrate for body shape and size. We performed a multisensory finger-stretch illusion that elongated the index finger. EBA volume and functional connectivity to the posterior parietal cortex are both related to the participants' susceptibility to the illusion. Taken together, these data suggest that EBA structure and connectivity encode body representation and body perception disturbances.
\end{abstract}

\section{KEYWORDS}

body image, body representation, extrastriate cortex, gray matter, magnetic resonance imaging, posterior parietal cortex, visual illusions

\section{1 | INTRODUCTION}

The representation of our body is essential for how we interact with our environment. These representations arise from multimodal sensory inputs, including visual, tactile, proprioceptive, interoceptive,

Massieh Moayedi and Nasim Noroozbahari contributed equally to this study. nociceptive, and motoric inputs (Longo \& Haggard, 2012). There are at least two proposed implicit body representations: body schema, which encodes the position of the body in space, and body image, which refers to the subjective experience of the size, shape, and features of the body (Head \& Holmes, 1911; Longo \& Haggard, 2012). Paillard (2005) differentiates body schema from body image by describing body schema as a vectorial coordinate map of sensorimotor

This is an open access article under the terms of the Creative Commons Attribution-NonCommercial-NoDerivs License, which permits use and distribution in any medium, provided the original work is properly cited, the use is non-commercial and no modifications or adaptations are made.

(c) 2021 The Authors. Human Brain Mapping published by Wiley Periodicals LLC. 
actions, centered on the body, which accounts for physical constraints (e.g., gravity). In contrast, body image is a dynamic map of the conscious experience within the spatial characteristics of the body (de Vignemont, 2010; Paillard, 2005). Whether body image and body schema are independent from each other and quite how they interact is yet to be established (Pitron, Alsmith, \& de Vignemont, 2018)-see de Vignemont (2010) and Pitron et al. (2018) for a comprehensive review. Nonetheless, considerably more research has been focused on the body schema, whereas the study of body image is still in its infancy (Pitron et al., 2018). This is because in most instances, body image relies on body schema, making it difficult to extrapolate specific neural and behavioral mechanisms that support violations of schemabased expectations (Pitron et al., 2018).

On this basis, the structural and functional neural network representation of body image requires further research. Both body and hand illusion studies provide evidence that body image representation is dynamic and plastic (Keizer, Smeets, Postma, van Elburg, \& Dijkerman, 2014; Keizer, van Elburg, Helms, \& Dijkerman, 2016). Here, we aim to determine the neural underpinnings of body image by leveraging this dynamism and plasticity using a body illusion.

Illusion studies are a reliable method to disturb body image under controlled conditions and are therefore powerful tools to investigate the neural mechanisms underlying body image (Blanke, 2012). For example, the Pinocchio illusion, and various derivatives of this unimodal illusion, have been used to induce body perception disturbances in healthy individuals (de Vignemont, Ehrsson, \& Haggard, 2005; Ehrsson, Kito, Sadato, Passingham, \& Naito, 2005). The illusory disruption of the body image can be reinforced with cross-modal illusions, which simultaneously manipulate two or more sensory channels (Meredith \& Stein, 1986; Shams, Wozny, Kim, \& Seitz, 2011). For example, the addition of a tactile stimulus can reinforce a visual illusion and subsequently enhance the robustness of the illusion, and thus susceptibility to the illusion. Integrating tactile and visual information requires multisensory processing and binding at several levels of the nervous system (Lemus, Hernandez, Luna, Zainos, \& Romo, 2010; Makin, Holmes, \& Ehrsson, 2008). Indeed, brain imaging studies of visuotactile illusions have identified the bilateral ventral premotor cortex (PMv), left posterior parietal cortex (PPC), and occipitotemporal areas (Cardini, Haggard, \& Ladavas, 2013; Limanowski \& Blankenburg, 2016; Limanowski, Lutti, \& Blankenburg, 2014; Makin et al., 2008; Mancini, Longo, lannetti, \& Haggard, 2011; Petkova et al., 2011) to be implicated in body representation. Unlike these previous illusion studies that modify body perception by targeting body schema, here, we specifically and exclusively modify body image.

The finger-stretch illusion is a robust visuotactile illusion in which participants experience alterations of their index finger in a computer-mediated augmented reality system with congruent sensory feedback from the experimenter (Newport et al., 2015); that is, the participant's finger appears to be elongating while the experimenter pulls on the tip of the finger, and shortening back to actual size while the experimenter pushes on the tip of the finger. An advantage of the finger-stretch illusion is that, unlike other visuotactile illusions (e.g., the rubber hand illusion [RHI]), the fingerstretch illusion is applied to the participant's own body through congruent manipulation of visual, proprioceptive and tactile stimuli (Preston \& Newport, 2011), rather than incorporating a nonbody object. This allows us to specifically target body image and not body schema, as the subject is not performing an action, nor interacting with the surrounding environment. Rather, they are passively viewing their finger change in shape and size. Importantly, ownership is not modified, and so multiple sensory channels are providing congruent information about the shape change of the finger.

We aim to use the finger-stretch illusion to identify the structural and functional network underpinnings of changes to body image representation. Here, we provide the first evidence for the extrastriate body area (EBA), a multisensory brain area, as the structural and functional neural substrate for body shape and size.

\section{2 | MATERIALS AND METHODS}

This study comprised two independent investigations: first, a behavioral study to determine whether a distal versus a proximal perspective affects the effectiveness and features of the body illusion; and second, an imaging study to identify the neural correlates of the illusion.

\section{3 | PARTICIPANTS}

\section{1 | Behavioral study (Experiment 1)}

Twelve right-handed adults ( 7 women, 5 men), aged (mean $\pm S D$ ) $23.6 \pm 2.3$ years were recruited for this study from the University of Nottingham, Nottingham, UK. Participants provided written informed consent to procedures reviewed and approved by the University of Nottingham ethics committee in line with the Declaration of Helsinki.

\subsection{Imaging study (Experiment 2)}

Twenty healthy adults were recruited from the University of Reading, Berkshire, UK. Participants provided written informed consent to procedures reviewed and approved by the University of Nottingham ethics committee in line with the Declaration of Helsinki, and were compensated for their time. Nineteen participants were scanned as one was excluded due to MRI contraindications. A further participant's dataset was excluded due to technical problems during the scan. Therefore, of the final sample of 18 participants (10 women, 8 men), aged $24.3 \pm 5.9$ years, 17 were right-handed, and one was left-handed.

\section{4 | MRI DATA ACQUISITION}

Functional brain images were collected on a $3 \mathrm{~T}$ MRI scanner (Magnetom Trio; Siemens, Erlangen, Germany) using a 32-channel 
head coil. For each participant, four runs of a 162 whole brain image time series ( $5 \mathrm{~min} 24 \mathrm{~s}$ ) were obtained using a gradient-echo, echoplanar scanning sequence (repetition time $(T R)=2 \mathrm{~s}$, echo time $(\mathrm{TE})=29 \mathrm{~ms}$, flip angle $=90^{\circ}$, GRAPPA $=2$, field of view $($ FOV $)=272 \mathrm{~mm}^{2}, 30$ axial slices, slice thickness $3.5 \mathrm{~mm}$, no gap, voxel size $=2.1 \times 2.1 \times 3.5 \mathrm{~mm}^{3}$ ). A high-resolution anatomical scan was also acquired (T1-weighted, three-dimensional magnetizationprepared rapid acquisition gradient echo sequence scan, TR $=2 \mathrm{~s}$, $\mathrm{TE}=2.99 \mathrm{~ms}$, FOV $=250 \mathrm{~mm}, 192$ sagittal slices, GRAPPA $=2$, voxel size $=1.0 \times 1.0 \times 1.0 \mathrm{~mm}^{3}$ ).

\section{5 | EXPERIMENTAL DESIGN}

\section{1 | Behavioral study}

We first sought to determine whether observing a finger-stretch illusion in an MRI scanner-that is, a distal setup rather than a proximal one-would affect the illusory experience. Participants underwent a finger-stretch illusion under two conditions (Figure 1): (1) proximal and (2) distal setup. In Setup 1, seated participants positioned their hand within the MIRAGE device, a mediated virtual reality system (University of Nottingham, Nottingham, UK). The Mirage device uses a camera and mirror arrangement where one can view real-time video images of their hand in the same perceived location as their actual hand. Instantaneous digital manipulations to the visual input give rise to a range of bodily illusions. We performed a finger stretch on the index finger of the left hand. This decision was based on the physical constraints in the MRI environment. Setup 2 was identical to Setup 1 with one key difference: the video image of the hand was on a screen two meters in front of the participant, thus removing the egocentric aspect of the illusion. This mimicked the setup inside the MRI scanner. Participants received two stretches per condition. Both setups used the same illusion on the participants' left index finger. Participants rated six statements about each stretch on an 11-point Likert scale, anchored at "not at all" and "extremely." The statements included two control statements: 1. "It felt like I had two left index fingers." 2. "My finger was getting hot," and four illusion susceptibility statements: 1 . "I feel like the finger I'm seeing belongs to me." 2. "I feel like I'm watching myself." 3. "I feel like my finger is longer than normal." 4. "It felt like my finger was really being stretched" (Figure 1c). The experimental questions have been used by the authors in previous manipulations of the index finger in a combined group of 91 participants (Perera, Newport, \& McKenzie, 2015; Perera, Newport, \& McKenzie, 2017).

\subsection{Imaging study}

All participants were naïve to the MIRAGE finger-stretch illusion (Newport et al., 2015; Preston \& Newport, 2011). As participants lay supine in the scanner, an MR-compatible camera in the MR-environment captured real-time digital images of the left index finger, to a computer in the control room. Participants viewed the image of the left index finger on a 32 in. fMRI compatible LCD screen (BOLDScreen, Cambridge Research Systems, Rochester, UK) through a mirror mounted onto the head coil. The experimenter stood next to the MRI table, holding the participant's left index throughout the MRI scan. The participants' remaining fingers were covered with a black, nonreflective cloth to be invisible in the image. The MIRAGE finger stretching illusion was run in the LabView Software package v 15.0 (National Instruments, Austin, TX) on an Apple MacBook Pro (Retina, 13-in., Early 2015, Apple, Cupertino, CA), running Windows 2008 (Microsoft, Seattle, WA). The illusion ("manipulation" condition) comprised of three distinct phases: index finger elongation (Figure 1a), maintenance of finger elongation, and shrinking the finger back to normal size. During finger elongation, participants observed their finger being lengthened while the experimenter simultaneously pulled gently on the distal tip of their finger. During maintenance, participants observed the experimenter holding the tip of the lengthened index finger. During shrinking, participants observed their index finger shrinking back to normal size, while the experimenter simultaneously pushed the tip of their finger. The experimenter was cued by an auditory signal on which tasks to perform. Each phase was $3 \mathrm{~s}$, and the whole illusion cycle took $9 \mathrm{~s}$. We also performed a control condition (nonmanipulation), where the same three illusion phases occurred, but without the finger being visually lengthened. Manipulations were identical across the group, and both participants and experimenters were blinded to the condition. During a $10 \mathrm{~s}$ period, $8 \mathrm{~s}$ after each illusion, participants rated the statement: "I felt like my finger was really being stretched" on a 6-point rating scale, where 0 represented "not at all" and 5 represented "extremely lengthened". The next illusion occurred $12 \mathrm{~s}$ after rating, with a pseudorandomized jitter of $0-3 \mathrm{~s}$, (rectangular distribution). The experiment utilized a block design, with four trials each of illusion and control conditions presented in a pseudo-randomized and counterbalanced order across four runs, for a total of 16 trials per condition.

\section{6 | DATA PREPROCESSING}

\section{1 | Ratings}

Each participant rated each trial of the finger stretch and the control conditions. These ratings were averaged across all trials for each illusion. Susceptibility scores were calculated by subtracting the control ratings from the finger-stretch illusion ratings.

\section{2 | Voxel-based morphometry}

To examine gray matter correlates of the susceptibility scores, we performed voxel-based morphometry (VBM) in the Statistical Parametric Mapping (v12; (http://www.fil.ion.ucl.ac.uk/spm/software/spm) DARTEL toolbox (Ashburner \& Friston, 2007). Briefly, preprocessing included setting the origin of the image at the anterior commissure of each subject, affine spatial normalization, tissue segmentation. Next, the various tissue classes were meaned, and were then aligned to create a template. Deformations from this template to each of the 
individual images were computed, and the template was then regenerated by applying the inverses of the deformations to the images and averaging. This procedure was repeated several times. The template was then normalized to Montreal Neurological Institute (MNI)-152 space. Finally, warped images were generated, and spatially smoothed with an $8 \mathrm{~mm}$ FWHM Gaussian kernel.

\section{(a) Finger-stretch illusion}
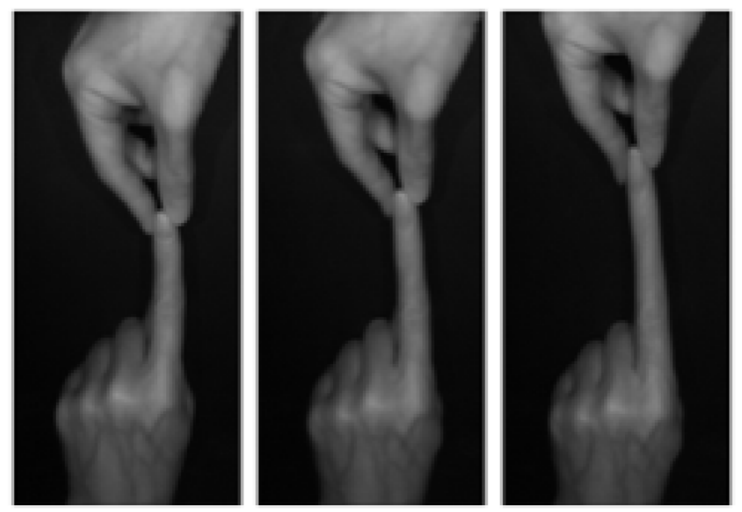

\section{(b) Experimental setups}
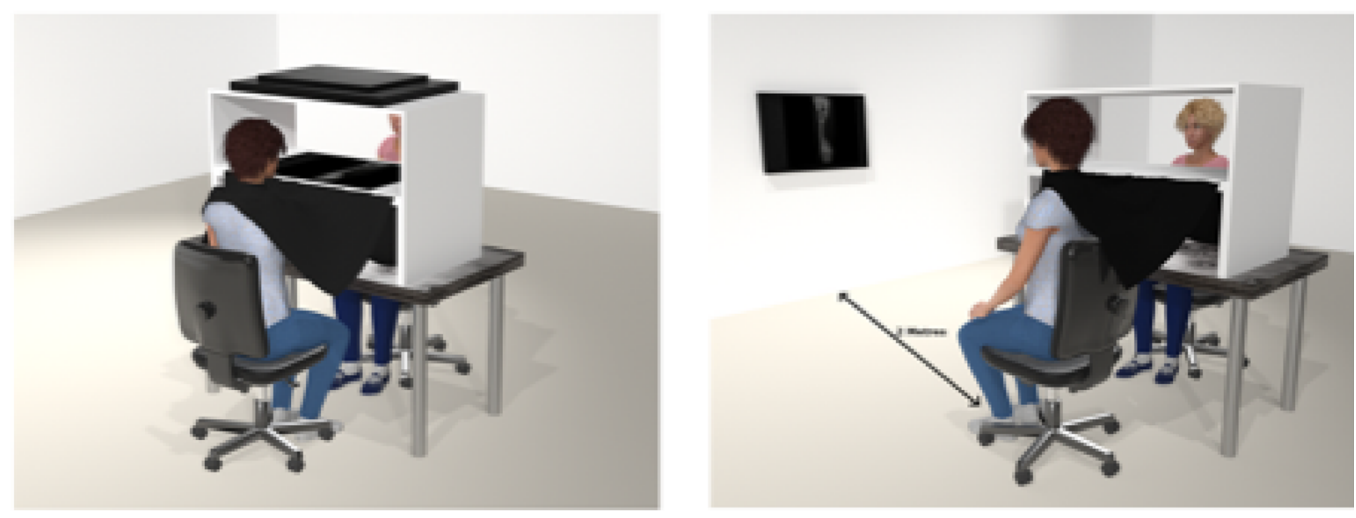

(c) Ratings for proximal and distal experimental setups

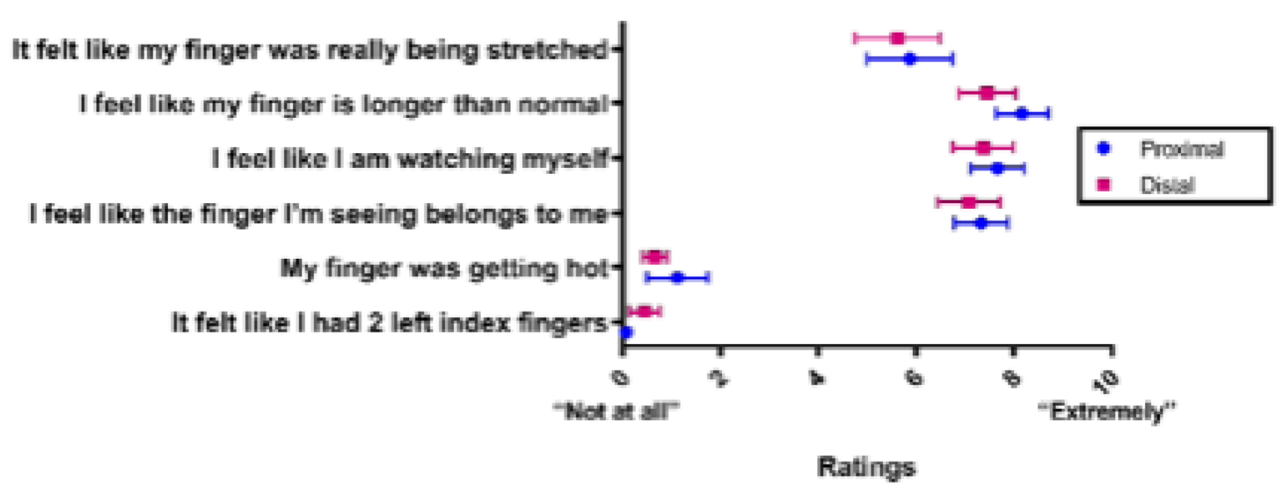

FIGURE 1 Behavioral experiment to determine whether the finger-stretch illusion can be performed within an MRI scanner with a different egocentric setup. (a) The finger-stretch illusion is a visuotactile illusion in which participants experience their index finger elongating in a computer-mediated augmented reality system with congruent sensory feedback from the experimenter. The progression of the illusion is shown from the left panel to the right. As the image of the finger is elongated, the experimenter pulls on the tip to add tactile feedback. (b) The illusion was tested in two experimental setups: (1) proximal (which is the original setup) and (2) distal $(n=12)$. The distal setup is similar to that which will be performed in the MRI. In the distal setup, the participant is watching a digital image of their finger undergoing the finger-stretch illusion. The screen is $2 \mathrm{~m}$ away from the participant. (c). Mean and SEM ratings of distal (magenta) and proximal (blue) setup are depicted. There were no significant differences between ratings for the proximal and distal setups, indicating that the feeling that the participant's own finger was stretched is similar and equally effective in the distal as the proximal setup (all related-samples Wilcoxon signed-rank tests $p>.05$. See Figure S1 and Table S1) 


\section{3 | Illusion task functional MRI analysis}

All imaging analysis was performed using FSL (FMRIB's Software Library, v.5.0, Oxford, UK) (Jenkinson, Beckmann, Behrens, Woolrich, \& Smith, 2012), unless otherwise indicated. Prior to statistical analysis, nonbrain structures were removed from each participant's structural images by Brain Extraction Tool (BET v.2.0). Preprocessing steps were performed using the Multivariate Exploratory Linear Optimized Decomposition into Independent Components (Beckmann \& Smith, 2004) toolbox. The first 5 volumes were removed for each participant to allow for signal equilibrium, and a high-pass filter cut off of $100 \mathrm{~s}(0.01 \mathrm{~Hz})$ was applied. Standard preprocessing, including motion correction using MCFLIRT (Jenkinson, Bannister, Brady, \& Smith, 2002), slice timing correction, spatial smoothing with a $5 \mathrm{~mm}$ (FWHM) Gaussian kernel was applied. The functional images were registered to the MNI-152 template. Data were then ICA-denoised by two independently trained raters (N. N. and A. Y.). To further denoise the dataset, we performed aCompCor procedures (Behzadi, Restom, Liau, \& Liu, 2007). Briefly, signals from the cerebrospinal fluid (CSF) and white matter (WM) were extracted. The CSF and WM masks were $5 \mathrm{~mm}$ spheres manually drawn in FSLview on the MNI-152 2-mm brain image. Principal components analysis was performed on each of the time courses in MATLAB v9.4 (MathWorks, Natick, MA), and the first five components were regressed out of the $\mathrm{FMRI}$ data for each participant.

\section{7 | STATISTICAL ANALYSIS}

\section{1 | Behavioral study}

Ratings from the different setups were tested for normality using the Shapiro-Wilk test. All behavioral statistical tests were performed using SPSS Statistics v27 for Mac (IBM Corp., Armonk, N.Y.). Across both setups, the illusion susceptibility ratings and the control ratings were not normally distributed. A nonparametric related-samples Wilcoxon signedrank test was used to compare each rating between the two setups. Significance was set to $p<.05$. Bonferroni correction was used to correct for six comparisons, which adjusted the significance to $p<.0083$.

\section{2 | Illusion task MRI ratings}

Illusion and control ratings were tested for normality using the Shapiro-Wilk test. Illusion ratings were normally distributed ( $p=.156$ ), while the control ratings were not $(p<.0001)$. As a result, a nonparametric related-samples Wilcoxon signed-rank test was used to compare average ratings between conditions. Significance was set to $p<.05$.

\section{3 | Voxel-based morphometry}

A whole brain, voxelwise statistical analysis was performed using the general linear model (GLM) to determine which gray matter regions correlated with susceptibility scores. The model included a regressor for group (to model the intercept), and demeaned susceptibility scores. Statistical images were thresholded at a cluster-corrected $p_{\mathrm{FWE}}<.05$, with a cluster-forming height threshold $p<.001$.

\section{4 | Illusion task functional MRI analysis}

A GLM analysis was carried out on the preprocessed and denoised data using FEAT (FMRI Expert Analysis Tool) version 6.00 of FSL (FMRIB's Software Library). Three different contrasts were modeled in the design matrix: finger-stretch, control, and fingerstretch > control. Motion parameters were not included in the model, as motion-related artifacts were corrected in preprocessing. A fixedeffect analysis was used to calculate a mean for each contrast across the four fMRI runs. Group-level analyses were performed using FLAME $1+2$ (FMRIB's Local Analysis of Mixed Effects) for each contrast. Statistical images were thresholded using a corrected-cluster $p<.05$ (cluster-forming height threshold $Z>3.1$ ). We also performed a conjunction analysis to identify brain regions that were activated by both the finger-stretch and control conditions (Nichols, Brett, Andersson, Wager, \& Poline, 2005). Briefly, the script identifies regions of significant overlap across two statistical images. Significance was set at a corrected-cluster $p<.05$ (cluster-forming height threshold $Z$ > 3.1).

\section{5 | Psychophysiological interaction analysis}

We sought to understand which set of brain regions were related to changes in body image. After identifying the regions of interest that displayed altered activation during the finger-stretch illusion task, a psychophysiological interaction (PPI) (Friston et al., 1997) analysis was performed to identify other voxels in the brain that displayed coupled activation with activity of these regions (https://fsl.fmrib.ox.ac.uk/fsl/ fslwiki/PPI). The advantage of using a PPI analysis is that it not only allows us to identify the brain network activated during the visual illusion, but correlations with the susceptibility ratings allow us to determine which brain regions are functionally connected during changes to the body image and relate to the susceptibility to that change. Seed ROls were the peak functional activations from the group-level analysis for the illusion > control contrast. We selected peak activations [all coordinates are reported in MNI152 space $(X, Y, Z)]$ within each ROI. Specifically, if a cluster spanned multiple ROls, then the highest statistical peak within the region was selected as the ROI. The coordinates for each ROI are: left PPC $(-46,-40,54)$, left EBA $(-42,-74,-2)$, left fusiform body areas (FBA; $-46,-58,-16)$, and left PMv $(-46$, $10,30)$; right PPC $(51,-26,38)$, right EBA $(48,-64,4)$, and right FBA $(48,-60,-12)$. Spheres (5 $\mathrm{mm}$ radius) centered around these peak coordinates were created and transformed to individual space using a linear transformation (FLIRT) to extract the time series (Jenkinson et al., 2002; Jenkinson \& Smith, 2001).

PPI analysis was initially performed on individual datasets by extracting deconvolved MR signals from each seed ROI. This 
extracted time course represents an approximation of neural activity which was centered and multiplied by a psychological factor (task condition). The PPI regressor was determined by the interaction between the time course and the psychological regressor (onset of the fingerstretch illusion). Both psychological and physiological regressors along with the PPI term were used in a GLM analysis. The GLM analysis resulted in a PPI connectivity map at the first level, which captured regions whose time series was correlated with the PPI regressor, above and beyond (orthogonal) to the main effect of the task.

Individual linear contrasts of PPI were subsequently grouped at the subject level (second level) using a fixed-effect analysis. At the group level, a mixed-effect analysis (FLAME 1 and 2) was performed to compare connectivity maps between participants. Statistical threshold was set at a corrected-cluster $p<.05$ (cluster-forming height threshold $Z>3.1$ ). Bonferroni correction was used to correct for the seven PPI analyses run, which led to an adjusted $p$-value of .007 .

Next, we investigated the relationship between resulting clusters of task-based functional connectivity during the illusion condition and the susceptibility ratings. We followed previously outlined methods (O'Reilly, Woolrich, Behrens, Smith, \& Johansen-Berg, 2012). Briefly, we extracted the connectivity values from the following PPI results: right EBA connectivity clusters: right PPC, right VIPFC, and R SMA; left EBA connectivity clusters: right PPC, right VIPFC, right SMA, and right FBA; left PMv connectivity cluster: right insular cortex; Right PPC connectivity cluster: left precuneus (see Supporting Information for left PMv and right PPC). A Shapiro-Wilk test was used to test the normality of connectivity values and susceptibility ratings. Susceptibility ratings were normally distributed $(p=.072)$ and connectivity values to the right EBA were normally distributed (all $p>.05$ ). Connectivity values for the left EBA were normally distributed (right PPC: $p=.325$; right SMA: $p=.167$, right FBA: $p=.981$ ), with the exception of the right VIPFC ( $p=.047)$. Pearson correlations were performed to test the relationship between right EBA connectivity values (to right PPC, vIPFC, and SMA) with susceptibility ratings. Spearman's correlations were performed to correlate left EBA connectivity values (to right PPC, SMA, FBA, and VIPFC) with susceptibility ratings. Significance was set to $p<.05$, Bonferroni-corrected. The critical $R^{2}$ value with $n=18$ for an adjusted $p<.017$ for the right EBA (Bonferroni corrected for three comparisons) is $R^{2}=.31$, and the $R^{2}$ critical value for an adjusted $p<.0125$ for the left EBA (Bonferroni-corrected for four comparisons) is $R^{2}=.33$.

\section{RESULTS}

\subsection{Behavioral study}

First, we sought to determine whether exposure to a finger-stretch illusion (see Figure 1a) in an MRI scanner with a different egocentric perspective to the usual direct and proximal perspective would affect the illusory experience. Thus, 12 healthy participants underwent the original finger-stretch illusion (with a proximal setup) and a modified finger-stretch illusion with distal setup akin to that used in the MRI environment (see Figure 1b). There were no significant differences in the experience of the two illusion setups (all Wilcoxon signed-rank tests $p>.05$; Figure 1c, Figure S1, and Table S1). Therefore, the MRI setup was not significantly different from the proximal setup.

\section{2 | Imaging study}

Eighteen participants experienced a finger-stretch illusion in a $3 \mathrm{~T}$ MRI scanner. During the illusion, the participant's left index finger was visually elongated with congruent tactile input. A control condition included all the same procedures without visual elongation. Participants provided trial-by-trial ratings of the extent to which they felt that their finger was actually being stretched in both conditions. Ratings were significantly greater during the illusion compared to the control condition ( $Z=3.528, p<.001)$, indicating that participants were susceptible to the illusion (Figure 2a).

\section{3 | Voxel-based morphometry}

We created a susceptibility score based on the average difference scores between the illusion and control trials for each participant. Individual differences in behavioral measures have been shown to be reflected in brain structure (Kanai \& Rees, 2011). To determine the structural gray matter underpinnings of individual differences in susceptibility, we performed a whole brain VBM analysis. We found that the bilateral EBA (part of the occipitotemporal cortex-OTC [Downing, Jiang, Shuman, \& Kanwisher, 2001]) volumes were positively correlated with susceptibility $\left(r^{2}=.74 ; p_{\mathrm{FWE}}<.05\right.$, with a cluster-forming height threshold $p<.001$; Figure 2b,c, and Table S2). In other words, the greater EBA volume, the more susceptible the participant was to the finger-stretch illusion.

\subsection{Whole brain activation during illusion}

We determined whole brain activation in response to the fingerstretch illusion, compared to the control condition, and found that multisensory brain areas-the bilateral occipitotemporal junction, in the area of the EBA and FBA, the bilateral PPC, the bilateral lateral occipital cortex, and left $\mathrm{PMv}$-showed greater activity during the illusion (Figure 3, Figures S2 and S4, Tables S3 and S4) (Carey, 2000; Lloyd, Shore, Spence, \& Calvert, 2003; McDonald, Teder-SÑlejÑrvi, \& Ward, 2001; Petkova et al., 2011). Notably, EBA did not show significant activation in the control condition (Figures S2 and S4, and Table S5). The activation of multisensory areas in response to the finger-stretch illusion is in line with other illusions (Limanowski et al., 2014; Limanowski \& Blankenburg, 2016). 
(a)

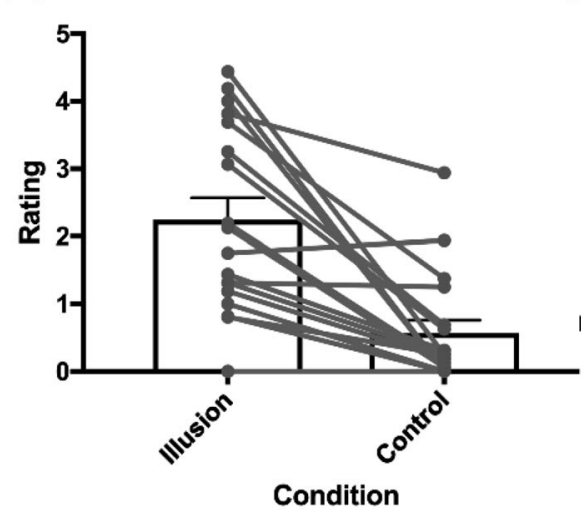

(b)

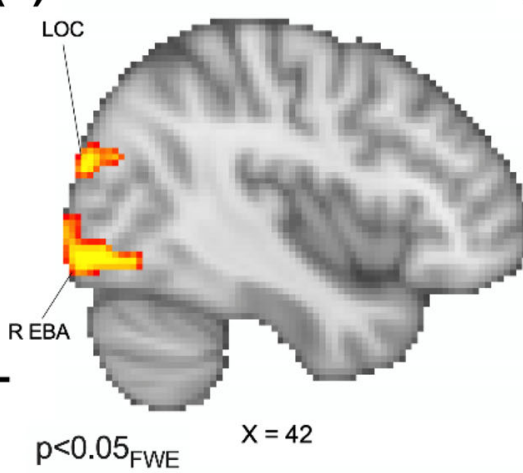

(c)

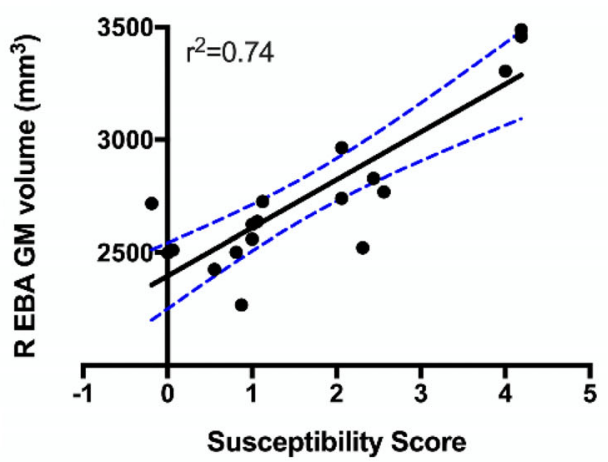

FIGURE 2 Susceptibility to illusion and neural correlates. (a) Individual participant ratings of the finger stretch illusion and control condition in the MRI scanner. These ratings (between 0 and 5) represent the susceptibility of the participants to the illusions. Mean ( \pm SE) of ratings to the statement "The extent to which you feel that your finger is actually being stretched" $(n=18)$. Participant ratings were based on 16 trials of each condition and were averaged across all trials for each condition. Susceptibility scores were calculated by subtracting the control ratings from the finger-stretch illusion ratings. Ratings for the illusion were significantly higher than the control condition ( $p=.00009$; Cohen's $d=1.19671$ ).

(b) Bilateral temporo-occipital gray matter volumes correlate with finger-stretch illusion susceptibility (the difference between the illusion and control conditions). Statistical images are cluster-corrected $p<.05$ family-wise error, with a cluster-forming height threshold of $p<.001$.

(c) Significant correlation of the right extrastriate body area gray matter volume with susceptibility ratings $\left(r^{2}=.74\right)$. Note that both EBA were significantly correlated with susceptibility, and the right EBA is shown for simplicity. Blue lines represent $95 \%$ confidence intervals. LOC, lateral occipital cortex; R EBA, right extrastriate body area

(a) Illusion > control

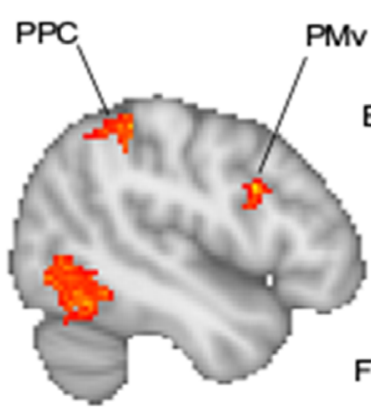

$X=-45$

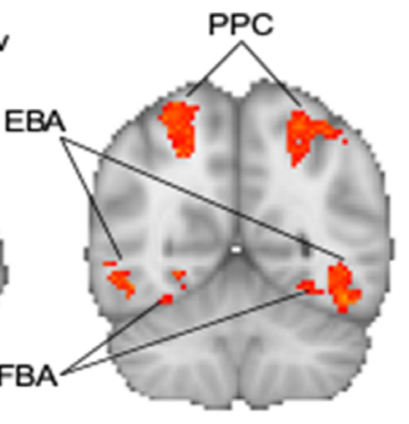

$Y=-55$

\section{(b) Illusion $\cap$ control}

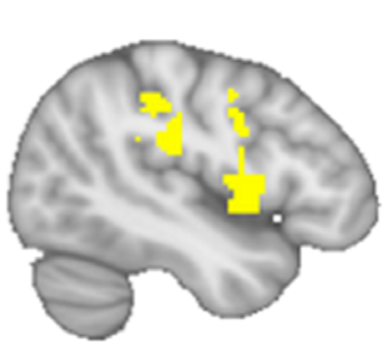

$x=-45$
EBA

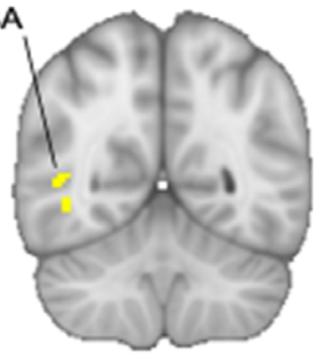

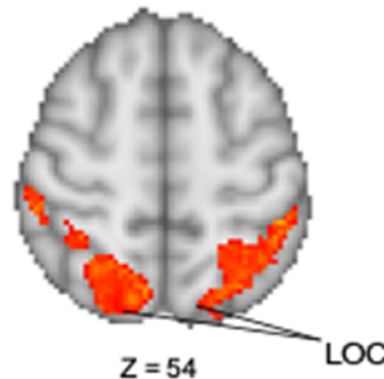

$Z=54$
5.57

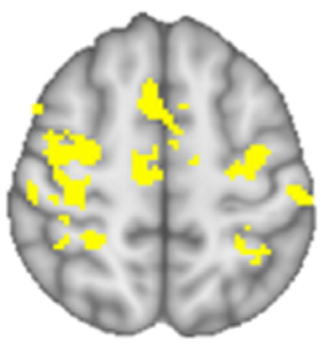

$Z=54$

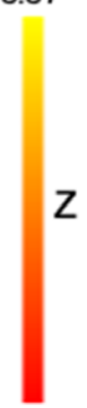

3.1

3.1

Z

3.1

FIGURE 3 Contrast and conjunction analyses between finger-stretch and control conditions. (a) Brain activations in response to the fingerstretch illusion compared to the control illusion $(n=18)$. (b) Conjunction map showing overlap of regions that show activation in both the fingerstretch illusion and the control illusion. Statistical images are cluster-corrected $p_{\mathrm{FWE}}<.05$ (cluster-forming height threshold $Z>3.1$ ). EBA, extrastriate body area; FBA, fusiform body area; LOC, lateral occipital cortex; PMv, ventral premotor cortex; PPC, posterior parietal cortex. Images are shown in radiological convention 


\subsection{Functional connectivity during illusion}

Following our structural and functional brain imaging findings of a relationship between the EBA and bodily illusion (Figures 2 and 3), we performed a PPI to determine which brain regions were functionally connected during the finger-stretch illusion. Given our a priori hypothesis about the role of the bilateral EBA in body image and susceptibility ratings, we report results for the remaining regions in Tables S9 and S10. We found that the left and right EBA were functionally connected to the right PPC, the right supplementary motor area, and the right ventrolateral prefrontal cortex (vIPFC; $p_{\mathrm{FWE}}<.05$, cluster-forming height threshold $Z>3.1$; Figure $4 a$, Figure S5, and

(a) Right EBA connectivity during illusion

Right EBA Seed

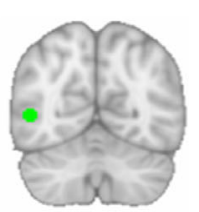

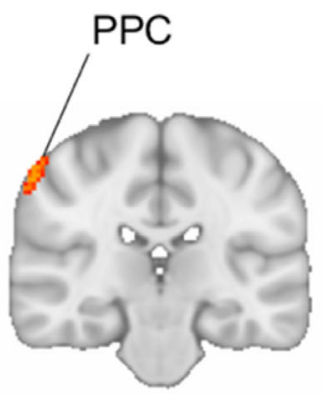

$\mathrm{Y}=-25$

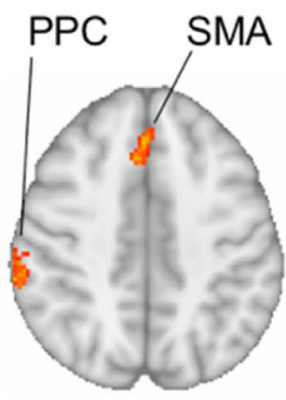

$Z=43$

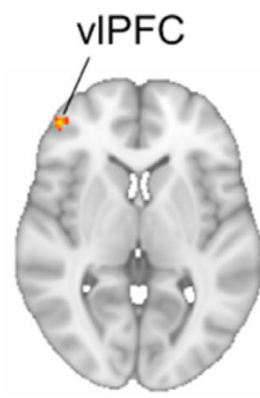

$Z=4$

Z - value

3.1

4.36

(b) Right EBA-Right PPC connectivity

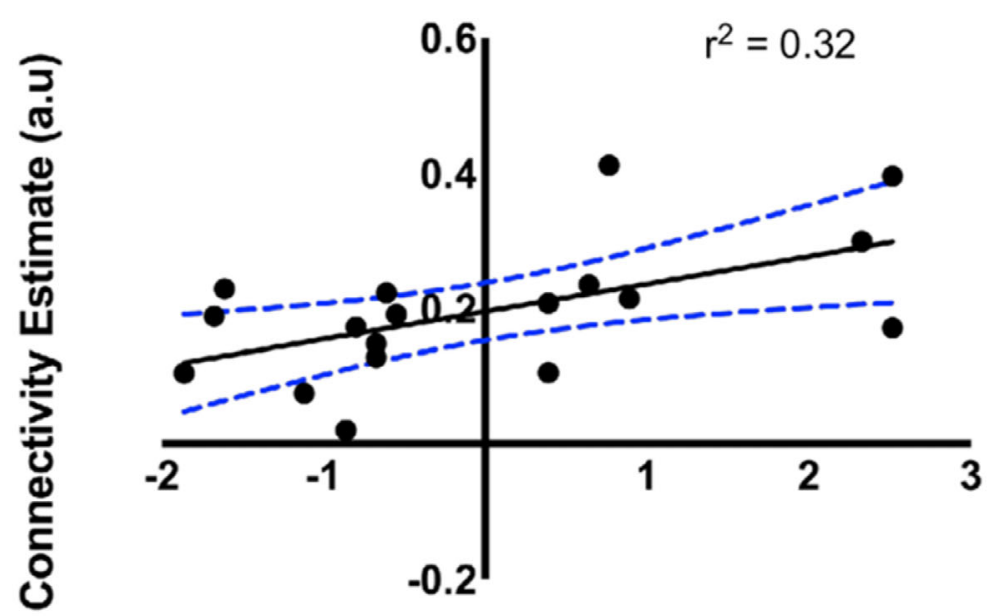

\section{Susceptibility}

FIGURE 4 Functional connectivity during illusion with susceptibility scores. (a) Psychophysiological interaction of the right extrastriate body area (EBA; shown in green) during the illusion condition. The EBA was significantly connected to the primary somatosensory cortex/posterior parietal cortex (S1/PPC), the supplementary motor area (SMA) and the ventrolateral prefrontal cortex (vIPFC). The statistical threshold includes a cluster corrected threshold of $p<.05$ ( $p<.007$, Bonferroni-corrected for psychophysiological interactions [PPIs] performed) using a cluster forming threshold $Z>3.1$. Note that bilateral EBA showed functional connectivity to these regions, but the right is shown for simplicity, and because it is contralateral to the illusion. (b) Positive correlation between right EBA-PPC task-based functional connectivity during the illusion condition and susceptibility scores $\left(R^{2}=.32, p<.017\right.$; Bonferroni-corrected $p<.05$ divided by three tests). Blue lines represent $95 \%$ confidence intervals. a.u., arbitrary units. Images are shown in radiological convention 
Tables S6 and S7). The left EBA was also functionally connected to the right FBA (Figure S5, Table S7). The left PMv was functionally connected to the right insular cortex $\left(p_{\mathrm{FWE}}<.05\right.$, cluster-forming height threshold $Z>3.1$; see Supporting Information, Table S9) and the right PPC showed decreased functional connectivity to the left precuneus $\left(p_{\mathrm{FWE}}<.05\right.$, cluster-forming height threshold $Z>3.1$; see Supporting Information, Table S10).

Most notably, we report that the connectivity between the right EBA and the right PPC was significantly correlated with susceptibility to the illusion $\left(r^{2}=.32, p=.014\right.$; Figure $4 b$, Table S8).

\section{9 | DISCUSSION}

We utilized a multimodal finger-stretch illusion to examine the behavioral and neural correlates of body image. We showed that the structure of the lateral occipitotemporal cortex, in the region of the EBA, was significantly correlated to how susceptible participants were to the illusion (Figure 2), and that multisensory regions were activated by the finger-stretch illusion (Figure 3), including bilateral lateral occipitotemporal cortex, in the region of the EBA (see Figures 3 and S4), PPC and PMv. Finally, we show that the task-based functional connectivity of the occipitotemporal cortex-the putative EBA-with the PPC is significantly correlated with susceptibility scores (Figure 4). Together, these multimodal data indicate that the lateral occipitotemporal cortex, in the region of the putative EBA, encodes body image perception.

Our findings further support the theory that body image representations are dynamic. Unlike the body schema, which depends on previous body experiences, the body image is free of physical constraints, as the shape and size of the body need not be realistic in order for perception and ownership to occur. This is supported by the results of our finger-stretch illusion, which stretched the index finger into a physically impossible size and shape, and yet participants still claimed body ownership to such manipulations. This suggested that body ownership was not constrained to the physical body or the experience of body, indicating that body image is indeed plastic. While alterations in EBA activity have been previously reported (Limanowski et al., 2014; Limanowski \& Blankenburg, 2015, 2016) little is known about the relationship between EBA gray matter volume and body image in healthy participants. We demonstrate that individual differences in body image perception correlate with bilateral EBA gray matter volume, suggesting that the EBA plays an important role in the extent of susceptibility to illusion and sensitivity to changes in body image. Our research is one of few studies that have depicted the association between the structure of EBA and body image. Previously, Suchan et al. (2010) reported the first evidence of altered EBA structure in women with anorexia nervosa. Interestingly, in those with anorexia nervosa, EBA gray matter density was negatively correlated to body size misjudgment. Our findings support the notion that structural alterations of EBA are associated with body image and perceived body shape.
We successfully demonstrated that body image perception can be manipulated by a finger-stretch illusion, which is in line with both hand and full body illusions that have been shown to improve the disturbed experience of body size in patients with anorexia nervosa (Keizer et al., 2016).

The EBA is an occipitotemporal region activated by congruent spatial and synchronous tactile stimuli (Limanowski \& Blankenburg, 2016, 2017). The EBA has been shown to respond to various stimuli, including human touch, action, and motion (Astafiev, Stanley, Shulman, \& Corbetta, 2004; Buchholz, David, Sengelmann, \& Engel, 2019; Downing, Peelen, Wiggett, \& Tew, 2006; Limanowski et al., 2014). Additionally, the EBA is involved in visual perception, the processing of human body parts, and the body as a whole (Downing et al., 2001; Suchan et al., 2010). Previous studies have identified selective disruption of body perception upon interference by transcranial magnetic stimulation (TMS) to the EBA (Calvo-Merino, Urgesi, Orgs, Aglioti, \& Haggard, 2010; Pitcher, Charles, Devlin, Walsh, \& Duchaine, 2009). Bilateral EBA activation would thus be expected to occur during the congruent visuotactile finger-stretch illusion and the control condition performed in the present study. The enhanced EBA activation in the illusion condition suggests that the EBA response is not only due to processing congruent visuotactile stimuli, but plays a direct role in body perception, more specifically in upper limb perception.

Enhanced PMv activity was observed during the illusion compared to the control condition. The PMv houses neurons with both visual and tactile receptive fields (Rizzolatti, Fogassi, \& Gallese, 2002) and is important for the detection of visuo-proprioceptive congruence (Downing \& Peelen, 2016; Limanowski \& Blankenburg, 2016). Our finding is in line with previous studies examining neural correlates of visual illusions, such as the RHI (Ehrsson, Spence, \& Passingham, 2004; Limanowski \& Blankenburg, 2015). In addition, the $\mathrm{PMv}$ is thought to relate to higher-level processing of upper limb representation and the surrounding (peri-personal) space (Makin et al., 2008). Studies have sought to elucidate the role of the PMv in the illusory experience and proprioception by utilizing a variety of tasks, such as arm positioning (Limanowski \& Blankenburg, 2016) and RHI manipulations (Ehrsson, Holmes, \& Passingham, 2005; Limanowski et al., 2014). The arm positioning task identifies brain regions active during congruent positioning of the participant's own unseen arm and a virtual realistic arm (Limanowski \& Blankenburg, 2016). The RHI paradigms manipulate either visual or tactile inputs, which control for the visualization of the rubber hand (somatic RHI) (Ehrsson, Holmes, \& Passingham, 2005) and the interference of the experimenter (automated RHI) (Limanowski et al., 2014), respectively. The PMv responds to the somatic RHI (Ehrsson, Holmes, \& Passingham, 2005) and arm positioning task (Limanowski \& Blankenburg, 2016), but not to the automated RHI (Limanowski et al., 2014), suggesting that this region is involved in processing tactile and proprioceptive inputs independent of human touch and action, as well as integrating congruent visual and proprioceptive information about arm position. The enhanced PMv activation 
observed in the present study therefore supports the role of the PMv in the integration of multisensory, visuotactile information in response to the finger-stretch illusion. This activation could support the role of the PMv in upper limb proprioception that is involved in body schema, by updating proprioceptive information upon receiving new visuotactile information.

Our second major finding shows significantly enhanced functional connectivity of the bilateral EBA to the PPC, supplementary motor area, and VIPFC during the finger-stretch illusion. Most notably, we report that the connectivity between the right EBA and the right PPC was significantly correlated with susceptibility to the illusion. This finding is consistent with Limanowski and Blankenburg (2017), who reported increased functional connectivity between the EBA and PPC during a visuo-proprioceptive RHI. However, the possibility of visual and proprioceptive integration occurring in the PPC rather than the EBA was not ruled out in their study. The PPC is thought to maintain a dynamic estimate of the perceptual representation of the body, in particular the hand region (Wolpert, Goodbody, \& Husain, 1998; Zimmer \& Macaluso, 2007), that can be updated through multisensory integration (Gentile, Petkova, \& Ehrsson, 2011; Petkova et al., 2011). Although other RHI studies have demonstrated increased EBA-PPC functional connectivity (Gentile, Guterstam, Brozzoli, \& Ehrsson, 2013; Limanowski \& Blankenburg, 2015), they were unable to attribute it to the visuoproprioceptive illusion. Rather than EBA-PPC connectivity, one RHI study demonstrated increased functional connectivity between the bilateral EBA and the primary somatosensory cortex (S1) (Limanowski et al., 2014). Inhibitory stimulation of the S1 hand region by repetitive TMS in healthy participants resulted in an overestimation of the perceived size of their hand (Giurgola, Pisoni, Maravita, Vallar, \& Bolognini, 2019), suggesting that the S1 plays a role not only in somatosensation, but in the perception of body size-which is in line with the fine representation of the hands in S1, compared to coarser representations in higher order brain regions, such as the PPC (Yau, Kim, Thakur, \& Bensmaia, 2016). A previous study proposed that the intraparietal sulcus (IPS), which separates the superior and inferior parietal lobules, minimizes mismatch between the incoming sensory information by integrating this visual information with tactile information. Specifically, the IPS integrates the somatosensory reference frame with the visual reference frame to minimize mismatch, and consequently increases its connectivity to the EBA (Limanowski \& Blankenburg, 2015). Taken together, the connectivity of the EBA to other parietal multisensory regions underlies body shape and size during multisensory illusions. In line with these previous findings, we demonstrate that EBA-PPC functional connectivity is related to a person's susceptibility to the finger-stretch illusion.

\section{0 | LIMITATIONS}

Our study has a few limitations that must be considered. Primarily, the study has a relatively small sample size. Future studies in larger, independent samples are required to reproduce our results.
Nonetheless, our study demonstrates the structure and function of the EBA in the neural underpinnings of body image using a fingerstretch illusion and rigorous statistical thresholds. Furthermore, our study is correlational in nature, and future studies should use causal techniques, such as neurostimulation, to confirm the role of the EBA in body image encoding.

\section{$11 \mid$ CONCLUSIONS}

Information from multiple sensory channels is integrated in higher cognitive areas to construct a body representation (Dijkerman \& de Haan, 2007). Specifically, the convergent somatosensory, proprioceptive and visual inputs to the EBA are integrated and underlie human body shape perception (Downing et al., 2001; Urgesi, Berlucchi, \& Aglioti, 2004). Our data suggest that the structural and functional connectivity of the EBA not only encodes the shape of body parts, but also how susceptible a participant is to disturbances of shape and size. Our structural and functional connectivity findings are the first to demonstrate the role of the EBA in perceiving changes to body shape using a multisensory illusion that manipulates the participant's own body in real time. More importantly, the structure of the EBA, and its functional connectivity to the PPC are correlated to the participant's susceptibility to changes in body shape-that is, body image. Body image disturbances-that is, body perception disturbances-have been reported in multiple disorders (Longo \& Haggard, 2012), including anorexia nervosa, bulimia nervosa, chronic pain (Lewis, Kersten, McCabe, McPherson, \& Blake, 2007; Lotze \& Moseley, 2007; Moseley, 2008; Moseley, Parsons, \& Spence, 2008; Sundermann, Flink, \& Linton, 2020), and somatoparaphrenia (Vallar \& Ronchi, 2009). Our results suggest that disorders of body image may be associated with changes in the structure of the EBA and its functional connections to other body encoding regions, such as the PPC. In summary, we have shown for the first time that the susceptibility to change in an individual's own body is correlated to the structure and function of the brain, specifically the structure of the EBA and functional connectivity between the EBA and the PPC. We found that the EBA is the structural and functional neural underpinning of body image using the fingerstretch illusion, which promotes multisensory integration using the participant's actual hand.

\section{ACKNOWLEDGMENT}

This study was entirely funded by a University of the West of England QR grant number 201415/31. Open Access funding enabled and organized by ProjektDEAL.

\section{DATA AVAILABILITY STATEMENT}

Data are available upon reasonable request.

\section{ETHICS STATEMENT}

Study 1: Procedures reviewed and approved by the University of Nottingham ethics committee. Study 2: Procedures reviewed and 
approved by the University of the West of England and the University of Reading ethics committees.

\section{ORCID}

Massieh Moayedi (D) https://orcid.org/0000-0002-7324-2540

Jennifer S. Lewis (D) https://orcid.org/0000-0002-6503-3749

\section{REFERENCES}

Ashburner, J., \& Friston, K. J. (2007). Voxel-based morphometry. In K. J. Friston (Ed.), Statistical parametric mapping the analysis of functional brain images (1st ed., pp. 92-98). Amsterdam: Elsevier.

Astafiev, S. V., Stanley, C. M., Shulman, G. L., \& Corbetta, M. (2004). Extrastriate body area in human occipital cortex responds to the performance of motor actions. Nature Neuroscience, 7, 542-548.

Beckmann, C. F., \& Smith, S. M. (2004). Probabilistic independent component analysis for functional magnetic resonance imaging. IEEE Transactions on Medical Imaging, 23, 137-152.

Behzadi, Y., Restom, K., Liau, J., \& Liu, T. T. (2007). A component based noise correction method (CompCor) for BOLD and perfusion based fMRI. Neurolmage, 37, 90-101.

Blanke, O. (2012). Multisensory brain mechanisms of bodily self-consciousness. Nature Reviews. Neuroscience, 13, 556-571.

Buchholz, V. N., David, N., Sengelmann, M., \& Engel, A. K. (2019). Belief of agency changes dynamics in sensorimotor networks. Scientific Reports, 9, 1995.

Calvo-Merino, B., Urgesi, C., Orgs, G., Aglioti, S. M., \& Haggard, P. (2010). Extrastriate body area underlies aesthetic evaluation of body stimuli. Experimental Brain Research, 204, 447-456.

Cardini, F., Haggard, P., \& Ladavas, E. (2013). Seeing and feeling for self and other: Proprioceptive spatial location determines multisensory enhancement of touch. Cognition, 127, 84-92.

Carey, D. P. (2000). Multisensory integration: Attending to seen and felt hands. Current Biology, 10, R863-R865.

de Vignemont, F. (2010). Body schema and body image-Pros and cons. Neuropsychologia, 48, 669-680.

de Vignemont, F., Ehrsson, H. H., \& Haggard, P. (2005). Bodily illusions modulate tactile perception. Current Biology, 15, 1286-1290.

Dijkerman, H. C., \& de Haan, E. H. (2007). Somatosensory processes subserving perception and action. The Behavioral and Brain Sciences, 30, 189-201 discussion 201-139.

Downing, P. E., Jiang, Y., Shuman, M., \& Kanwisher, N. (2001). A cortical area selective for visual processing of the human body. Science, 293, 2470-2473.

Downing, P. E., \& Peelen, M. V. (2016). Body selectivity in occipitotemporal cortex: Causal evidence. Neuropsychologia, 83, 138-148.

Downing, P. E., Peelen, M. V., Wiggett, A. J., \& Tew, B. D. (2006). The role of the extrastriate body area in action perception. Social Neuroscience, 1, 52-62.

Ehrsson, H. H., Holmes, N. P., \& Passingham, R. E. (2005). Touching a rubber hand: Feeling of body ownership is associated with activity in multisensory brain areas. The Journal of Neuroscience, 25, 10564-10573.

Ehrsson, H. H., Kito, T., Sadato, N., Passingham, R. E., \& Naito, E. (2005). Neural substrate of body size: Illusory feeling of shrinking of the waist. PLoS Biology, 3, e412.

Ehrsson, H. H., Spence, C., \& Passingham, R. E. (2004). That's my hand! Activity in premotor cortex reflects feeling of ownership of a limb. Science, 305, 875-877.

Friston, K. J., Buechel, C., Fink, G. R., Morris, J., Rolls, E., \& Dolan, R. J. (1997). Psychophysiological and modulatory interactions in neuroimaging. Neurolmage, 6, 218-229.

Gentile, G., Guterstam, A., Brozzoli, C., \& Ehrsson, H. H. (2013). Disintegration of multisensory signals from the real hand reduces default limb self-attribution: An fMRI study. The Journal of Neuroscience, 33, 13350-13366.

Gentile, G., Petkova, V. I., \& Ehrsson, H. H. (2011). Integration of visual and tactile signals from the hand in the human brain: An FMRI study. Journal of Neurophysiology, 105, 910-922.

Giurgola, S., Pisoni, A., Maravita, A., Vallar, G., \& Bolognini, N. (2019). Somatosensory cortical representation of the body size. Human Brain Mapping, 40, 3534-3547.

Head, H., \& Holmes, G. (1911). Sensory disturbances from cerebral lesions. Brain, 34, 102-254.

Jenkinson, M., Bannister, P., Brady, M., \& Smith, S. (2002). Improved optimization for the robust and accurate linear registration and motion correction of brain images. Neurolmage, 17, 825-841.

Jenkinson, M., Beckmann, C. F., Behrens, T. E., Woolrich, M. W., \& Smith, S. M. (2012). Fsl. Neurolmage, 62, 782-790.

Jenkinson, M., \& Smith, S. (2001). A global optimisation method for robust affine registration of brain images. Medical Image Analysis, 5, 143-156.

Kanai, R., \& Rees, G. (2011). The structural basis of inter-individual differences in human behaviour and cognition. Nature Reviews. Neuroscience, 12, 231-242.

Keizer, A., Smeets, M. A., Postma, A., van Elburg, A., \& Dijkerman, H. C. (2014). Does the experience of ownership over a rubber hand change body size perception in anorexia nervosa patients? Neuropsychologia, 62, 26-37.

Keizer, A., van Elburg, A., Helms, R., \& Dijkerman, H. C. (2016). A virtual reality full body illusion improves body image disturbance in anorexia nervosa. PLoS One, 11, e0163921.

Lemus, L., Hernandez, A., Luna, R., Zainos, A., \& Romo, R. (2010). Do sensory cortices process more than one sensory modality during perceptual judgments? Neuron, 67, 335-348.

Lewis, J. S., Kersten, P., McCabe, C. S., McPherson, K. M., \& Blake, D. R. (2007). Body perception disturbance: A contribution to pain in complex regional pain syndrome (CRPS). Pain, 133, 111-119.

Limanowski, J., \& Blankenburg, F. (2015). Network activity underlying the illusory self-attribution of a dummy arm. Human Brain Mapping, 36, 2284-2304.

Limanowski, J., \& Blankenburg, F. (2016). Integration of visual and proprioceptive limb position information in human posterior parietal, premotor, and extrastriate cortex. The Journal of Neuroscience, 36, 2582-2589.

Limanowski, J., \& Blankenburg, F. (2017). Posterior parietal cortex evaluates visuoproprioceptive congruence based on brief visual information. Scientific Reports, 7, 16659.

Limanowski, J., Lutti, A., \& Blankenburg, F. (2014). The extrastriate body area is involved in illusory limb ownership. Neurolmage, 86, 514-524.

Lloyd, D. M., Shore, D. I., Spence, C., \& Calvert, G. A. (2003). Multisensory representation of limb position in human premotor cortex. Nature Neuroscience, 6, 17-18.

Longo, M. R., \& Haggard, P. (2012). Implicit body representations and the conscious body image. Acta Psychologica, 141, 164-168.

Lotze, M., \& Moseley, G. L. (2007). Role of distorted body image in pain. Current Rheumatology Reports, 9, 488-496.

Makin, T. R., Holmes, N. P., \& Ehrsson, H. H. (2008). On the other hand: Dummy hands and peripersonal space. Behavioural Brain Research, 191, 1-10

Mancini, F., Longo, M. R., lannetti, G. D., \& Haggard, P. (2011). A supramodal representation of the body surface. Neuropsychologia, 49, 1194-1201.

McDonald, J. J., Teder-SÑlejÑrvi, W. A., \& Ward, L. M. (2001). Multisensory integration and crossmodal attention effects in the human brain. Science, 292, 1791a.

Meredith, M. A., \& Stein, B. E. (1986). Visual, auditory, and somatosensory convergence on cells in superior colliculus results in multisensory integration. Journal of Neurophysiology, 56, 640-662. 
Moseley, G. L. (2008). I can't find it! Distorted body image and tactile dysfunction in patients with chronic back pain. Pain, 140, 239-243.

Moseley, G. L., Parsons, T. J., \& Spence, C. (2008). Visual distortion of a limb modulates the pain and swelling evoked by movement. Current Biology, 18, R1047-R1048.

Newport, R., Auty, K., Carey, M., Greenfield, K., Howard, E. M., Ratcliffe, N., ... Themelis, K. (2015). Give it a tug and feel it grow: Extending body perception through the universal nature of illusory finger stretching. Iperception, 6, 2041669515599310.

Nichols, T., Brett, M., Andersson, J., Wager, T., \& Poline, J. B. (2005). Valid conjunction inference with the minimum statistic. Neurolmage, 25, 653-660.

O'Reilly, J. X., Woolrich, M. W., Behrens, T. E., Smith, S. M., \& JohansenBerg, H. (2012). Tools of the trade: Psychophysiological interactions and functional connectivity. Social Cognitive and Affective Neuroscience, 7, 604-609.

Paillard, J. (2005). Vectorial versus configural encoding of body space: A neural basis for a distinction between body schema and body image. In H. de Preester \& V. Knockaert (Eds.), Body image and body schema: Interdisciplinary perspectives on the body (pp. 89-109). Amsterdam, The Netherlands: John Benjamins Publishing Company.

Perera, A. T., Newport, R., \& McKenzie, K. J. (2017). Changing hands: Persistent alterations to body image following brief exposure to multisensory distortions. Experimental Brain Research, 235, 1809-1821.

Perera, T. M. A., Newport, R., \& McKenzie, K. J. (2015). Multisensory distortions of the hand have differential effects on tactile perception. Experimental Brain Research, 233, 3153-3161.

Petkova, V. I., Bjornsdotter, M., Gentile, G., Jonsson, T., Li, T. Q., \& Ehrsson, H. H. (2011). From part- to whole-body ownership in the multisensory brain. Current Biology, 21, 1118-1122.

Pitcher, D., Charles, L., Devlin, J. T., Walsh, V., \& Duchaine, B. (2009). Triple dissociation of faces, bodies, and objects in extrastriate cortex. Current Biology, 19, 319-324.

Pitron, V., Alsmith, A., \& de Vignemont, F. (2018). How do the body schema and the body image interact? Consciousness and Cognition, 65, 352-358.

Preston, C., \& Newport, R. (2011). Analgesic effects of multisensory illusions in osteoarthritis. Rheumatology (Oxford), 50, 2314-2315.

Rizzolatti, G., Fogassi, L., \& Gallese, V. (2002). Motor and cognitive functions of the ventral premotor cortex. Current Opinion in Neurobiology, 12, 149-154.
Shams, L., Wozny, D. R., Kim, R., \& Seitz, A. (2011). Influences of multisensory experience on subsequent unisensory processing. Frontiers in Psychology, 2, 264.

Suchan, B., Busch, M., Schulte, D., Gronemeyer, D., Herpertz, S., \& Vocks, S. (2010). Reduction of gray matter density in the extrastriate body area in women with anorexia nervosa. Behavioural Brain Research, 206, 63-67.

Sundermann, O., Flink, I., \& Linton, S. J. (2020). My body is not working right: A cognitive behavioral model of body image and chronic pain. Pain, 161, 1136-1139.

Urgesi, C., Berlucchi, G., \& Aglioti, S. M. (2004). Magnetic stimulation of extrastriate body area impairs visual processing of nonfacial body parts. Current Biology, 14, 2130-2134.

Vallar, G., \& Ronchi, R. (2009). Somatoparaphrenia: A body delusion. A review of the neuropsychological literature. Experimental Brain Research, 192, 533-551.

Wolpert, D. M., Goodbody, S. J., \& Husain, M. (1998). Maintaining internal representations: The role of the human superior parietal lobe. Nature Neuroscience, 1, 529-533.

Yau, J. M., Kim, S. S., Thakur, P. H., \& Bensmaia, S. J. (2016). Feeling form: The neural basis of haptic shape perception. Journal of Neurophysiology, 115, 631-642.

Zimmer, U., \& Macaluso, E. (2007). Processing of multisensory spatial congruency can be dissociated from working memory and visuospatial attention. The European Journal of Neuroscience, 26, 16811691.

\section{SUPPORTING INFORMATION}

Additional supporting information may be found online in the Supporting Information section at the end of this article.

How to cite this article: Moayedi M, Noroozbahari N, Hadjis G, et al. The structural and functional connectivity neural underpinnings of body image. Hum Brain Mapp. 2021; 42:3608-3619. https://doi.org/10.1002/hbm.25457 